\title{
Limitações dos Modelos de Aceitação da Tecnologia: um ensaio sob uma perspectiva crítica
}

\section{Boundaries of Technology Acceptance Models: an essay from a critical perspective}

Jéssica Vivianne da Cunha Silva de Brito*1, Anatália Saraiva Martins Ramos ${ }^{1}$

${ }^{1}$ Programa de Pós-Graduação em Administração, Universidade Federal do Rio Grande do Norte, RN, Brasil.

\begin{tabular}{|c|c|}
\hline I NFO A R T I GO & RES U MO \\
\hline $\begin{array}{l}\text { Palavras-chave: } \\
\text { Modelo de Aceitação da } \\
\text { Tecnologia, } \\
\text { TAM, } \\
\text { UTAUT, } \\
\text { TRA, } \\
\text { TPB. }\end{array}$ & $\begin{array}{l}\text { O presente trabalho tem como objetivo fazer uma análise crítica acerca dos } \\
\text { modelos teóricos que se propõem a explicar as variáveis que influenciam a } \\
\text { intenção de uso de uma tecnologia por parte do usuário. Após a exposição da } \\
\text { problemática, é realizada uma breve exposição das principais teorias de } \\
\text { aceitação da tecnologia existentes, quais sejam: a TRA, a TPB, o TAM (com } \\
\text { seus desdobramentos TAM } 2 \text { e TAM 3) e a UTAUT, pontuando-se os fatores } \\
\text { que não permitem a essas teorias responder contundentemente quais variáveis } \\
\text { influenciam o uso das tecnologias. Por fim, acredita-se que os modelos } \\
\text { apresentados são eficazes no que se propõem e que as limitações existentes } \\
\text { são decorrentes da complexidade do fenômeno que eles buscam tratar, sendo } \\
\text { pouco provável a existência de um modelo ótimo capaz de responder de forma } \\
\text { completa a questões sobre quais os fatores que influenciam o uso de uma } \\
\text { tecnologia da informação. }\end{array}$ \\
\hline ARTICLE INFO & A B S T R ACT \\
\hline $\begin{array}{l}\text { Keywords: } \\
\text { technology acceptance } \\
\text { model, } \\
\text { TAM, } \\
\text { UTAUT, } \\
\text { TRA, } \\
\text { TPB. }\end{array}$ & $\begin{array}{l}\text { The present work aims to make a critical analysis about the theoretical models } \\
\text { that propose to explain the variables that influence the intention of using a } \\
\text { technology by the user. After the exposure of the problem, a brief exposition } \\
\text { of the main theories of acceptance of the existing technology is presented, } \\
\text { namely: TRA, TPB, TAM (with its ramifications TAM } 2 \text { and TAM 3) and } \\
\text { UTAUT, talking about the factors that do not allow these theories to answer } \\
\text { strongly which variables influence the use of technologies. Finally, it is } \\
\text { believed that the models presented are effective in their purpose and that the } \\
\text { existing limitations are due to the complexity of the phenomenon they seek to } \\
\text { treat and it is unlikely that there is an optimal model that can fully answer } \\
\text { questions about which factors influence the use of an information technology. }\end{array}$ \\
\hline
\end{tabular}

\section{Introdução}

O debate acerca dos fatores que influenciam o uso ou a intenção de uso de uma tecnologia da informação não é algo recente. Existe um grande número de modelos que buscam explicar esse fenômeno (Lai, 2017; Scherer, Siddiq \& Tondeur, 2019). Todavia, observando o estado da arte, é possível dizer que os modelos existentes não conseguem identificar com precisão todas as variáveis que influenciam o processo de decisão quanto ao uso ou não de uma tecnologia da informação (Venkatesh, Morris, Davis \& Davis, 2003).

\footnotetext{
${ }^{*}$ Correspondência para autor:

brito.jessicacunha@gmail.com (Brito, J. V. da C. S. de) (ORCID: 0000-0002-6814-492X), anataliasaraiva@gmail.com (Ramos, A. S. M.).

http://dx.doi.org/10.21714/1679-18272019v17Esp.p210-220

1679-1827 C 2019 Gest@o.org.
} 
Isso ocorre, dentre outros motivos, em função de que nesse espectro de análise, o indivíduo é o ator principal do processo de decisão e, assim sendo, devem ser observados fatores excepcionais relacionados ao comportamento e às perspectivas do usuário enquanto indivíduo dotado de valores, crenças e conhecimentos próprios (Tavares $\&$ Costa, 2008). Tais fatores por algum tempo ficavam fora do escopo do processo de desenvolvimento de uma tecnologia em razão da vigência de um paradigma tecnicista no qual as tecnologias eram privilegiadas em detrimento ao indivíduo, que se caracterizava apenas como um agente passivo, que precisava se adaptar à tecnologia (Bouwman, Van Den Hooff, Van De Wijngaert \& Van Dijk, 2005).

Com o avanço da análise acerca dessas questões, novos modelos analíticos foram criados e passaram a incorporar essa subjetividade inerente aos indivíduos (Tavares \& Costa, 2008). Aspectos particulares ligados diretamente às expectativas e ao comportamento dos indivíduos começaram a incrementar os modelos, que melhoraram a qualidade de ajuste e a capacidade de explicar os fenômenos. Contudo, verifica-se a ocorrência de fatores que podem influenciar o uso das tecnologias pelos indivíduos e que não estão devidamente contemplados pelos modelos existentes. Aponta-se necessidades de status e urgência como algumas dessas variáveis que podem influenciar o uso das tecnologias pelos indivíduos, mas não estão diretamente contempladas pelos modelos vigentes.

Neste trabalho, pontua-se que os modelos existentes não são inadequados ou equivocados, apenas que eles não contemplam todos os fatores que influenciam a intenção de uso de uma tecnologia, isto é, não há um caminho padronizado sobre o qual o planejamento de uma tecnologia possa ser traçado de modo que a intenção de uso por parte dos usuários seja inequívoca.

Modelos sintéticos, como, por exemplo, o Modelo de Aceitação da Tecnologia (Technology Acceptance Model - TAM), a Teoria da Ação Racional (Theory of Reasoned Action - TRA) ou a Teoria do Comportamento Planejado (Theory of Planned Behavior - TPB), só conseguem explicar aproximadamente 50\% dos fatores que interferem na decisão de uso de uma tecnologia (Venkatesh et al., 2003).

O avanço do debate fez surgir a Teoria Unificada de Aceitação e Uso da Tecnologia - UTAUT, que é um modelo resultante de testes de modelos anteriores, cujos construtos foram validados por aplicações empíricas realizadas por professores especialistas no tema, como Venkatesh et al. (2003), à qual teria sido concebida com o intuito de ser uma espécie de "modelo ideal". Todavia, mesmo esse modelo mais complexo só consegue responder cerca de $70 \%$ da questão tratada. Apesar desse índice ser considerado elevado, há poucos estudos que se dedicam a validar e explorar o desempenho desse modelo (Williams, Rana \& Dwivedi, 2015), havendo, inclusive, aplicações que denotam que o TAM e a TPB apresentam níveis de resposta mais elevados do que os alcançados pela UTAUT (Rahman, Lesch, Horrey \& Strawderman, 2017).

Assim, emerge a reflexão sobre os porquês desses modelos não conseguirem um nível mais elevado de resposta para a intenção de uso de uma tecnologia. Como dito anteriormente, existem inúmeras tentativas de compreensão desse fenômeno, autores como Davis $(1989 ; 2000)$ e Venkatesh $(2000 ; 2003)$ dedicaram-se à pesquisa sobre tais fatores, mas não conseguiram finalizar um modelo cujo nível de assertividade seja mais elevado que o UTAUT (Venkatesh et al., 2003).

Assim sendo, busca-se com este trabalho fazer uma análise crítica acerca dos modelos teóricos existentes que se propõem a explicar as variáveis que influenciam a intenção de uso de uma tecnologia por parte do usuário. Para tanto, serão apresentadas as teorias de maior relevância no campo teórico, estabelecendo-se de forma pontual comentários sobre os motivos que fazem com que cada um dos modelos relatados não consiga responder de forma contundente a questão em discussão.

Destaca-se por fim que a ideação deste trabalho é resultado de uma análise crítica, não sendo objetivo deste ensaio teórico julgar o mérito ou a qualidade dos modelos analisados. Tenciona-se tão somente propor uma reflexão sobre as lacunas existentes em cada modelo, o que - conforme já discutido - se deve à complexidade e à pluralidade características do fenômeno estudado.

Outro ponto relevante a se destacar é o fato de que há na literatura nacional poucos trabalhos que se dedicam a fazer uma análise teórica sob uma perspectiva crítica dos modelos de aceitação da tecnologia existentes. Além disso, a maioria dos estudos existentes se prestam a realizar uma análise de um modelo específico (Meirelles \& Longo, 2014; Bobsin, Visentini \& Rech, 2009) ou uma comparação entre dois ou três previamente definidos (Carvalho, Freitas, Ramos, Nascimento \& Ferreira, 2013; Silva \& Dias, 2007).

Assim, este trabalho se caracteriza como um ensaio teórico, cujos pressupostos metodológicos possibilitam a defesa de ideias de maneira fundamentada, porém livre das rígidas formalidades exigidas para determinadas 
espécies do fazer científico (Boava, Macedo \& Sette, 2012), o que possibilita a realização de uma análise crítica mais ampla e holística sobre o fenômeno estudado.

\section{Análise Crítica das Teorias de Aceitação da Tecnologia}

Nesta seção são apresentados brevemente os modelos: Teoria da Ação Racional, Teoria do Comportamento Planejado, Modelo de Aceitação da Tecnologia - e versões posteriores - e a Teoria Unificada de Aceitação e Uso de Tecnologia. A ideia é que a partir da explicação de cada um deles seja possível realizar uma análise crítica sobre o nível de resposta deles à questão principal que norteia esse trabalho.

Para constituição deste estudo, foram selecionadas as teorias mais abordadas no âmbito dos trabalhos sobre aceitação e uso de tecnologias da informação (Lai, 2017). Estas teorias são as mais utilizadas em função de possibilitarem a análise deste fenômeno em diferentes contextos e configurações (IDEM).

\subsection{Teoria da Ação Racional - TRA}

A Teoria da Ação Racional (ou em inglês, Theory of Reasoned Action) defende que o comportamento de um indivíduo é determinado por sua intenção de comportamento, ou seja, pela intenção que o indivíduo possui de realizar determinado comportamento, e essa intenção de comportamento, por sua vez, é influenciada pela atitude do indivíduo e pelas normas subjetivas que exercem influência sobre ele (Fishbein \& Ajzen, 1975). A Figura 1 explicita a relação causal defendida pela teoria.

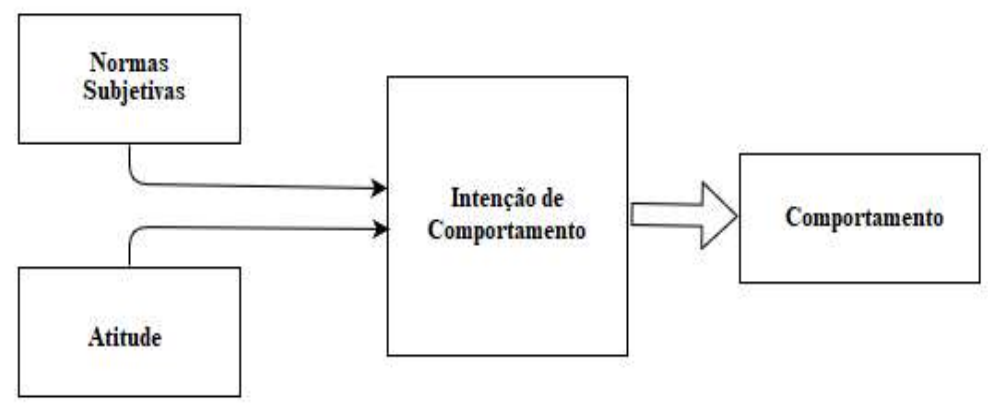

Figura 1 - Teoria da Ação Racional.

Fonte: adaptado de Fishbein e Ajzen (1975).

Conforme os pressupostos da teoria, a atitude diz respeito aos sentimentos positivos ou negativos que o indivíduo possui quanto à realização de determinado comportamento. Esses sentimentos seriam resultados das avaliações que ele realiza sobre as crenças que possui quanto às consequências que o desempenho de certo comportamento irá gerar para si, bem como da avaliação da conveniência dessas consequências. No que concerne às normas subjetivas, evidencia-se que se trata da percepção do indivíduo quanto à opinião de pessoas - que ele julga relevantes - sobre se ele deve ou não executar o comportamento analisado (Fishbein \& Ajzen, 1975).

Analisando-se a teoria à luz da perspectiva da adoção de tecnologias da informação, observa-se que a TRA se fundamenta na ideia, que os indivíduos definem os seus comportamentos a partir de uma análise racional, a qual é resultado de ponderações sobre os fatores que exercem influência sobre eles. Todavia, em se tratando da decisão de uso quanto a uma tecnologia, é improvável conceber que essa decisão é feita de maneira objetiva e considerando apenas a avaliação do usuário quanto à decisão de uso. É possível que muitos outros fatores influenciem esse processo de decisão e que a decisão do indivíduo não se origine exclusivamente de um julgamento racional.

Assim sendo, afora outras críticas relativas à capacidade explicativa da TRA (Davis, Bagozzi \& Warshaw, 1989), as quais não são objeto de análise deste trabalho, a Teoria da Ação Racional se mostra insuficiente para determinar os fatores que impactam a decisão de uso de uma tecnologia por parte do indivíduo à medida que não considera variáveis subjetivas que provavelmente influenciam a decisão deste.

\subsection{Teoria do Comportamento Planejado - TPB}

A Teoria do Comportamento Planejado (em inglês, Theory of Planned Behavior - TPB) é um desdobramento da Teoria da Ação Racional, cujo objetivo foi agregar aos construtos da TAR um novo fator com potencial para 
explicar a intenção de comportamento dos indivíduos: o controle comportamental percebido. Essa variável diz respeito à facilidade percebida ou à dificuldade de realizar o comportamento. Ela foi inserida no modelo sob a perspectiva de auxiliar a análise das crenças que o indivíduo possui quanto à disponibilidade de recursos e oportunidades e à existência de dificuldades para realização do comportamento em questão (Ajzen, 2011).

De acordo com o autor da teoria, Ajzen (1991), a teoria foi planejada para propiciar a previsão e a explicação do comportamento humano diante de situações específicas, sendo fundamentada pelo pressuposto que o comportamento dos indivíduos é baseado na intenção que eles apresentam de efetivar tal comportamento. A Figura 2 aclara as relações defendidas pela teoria.

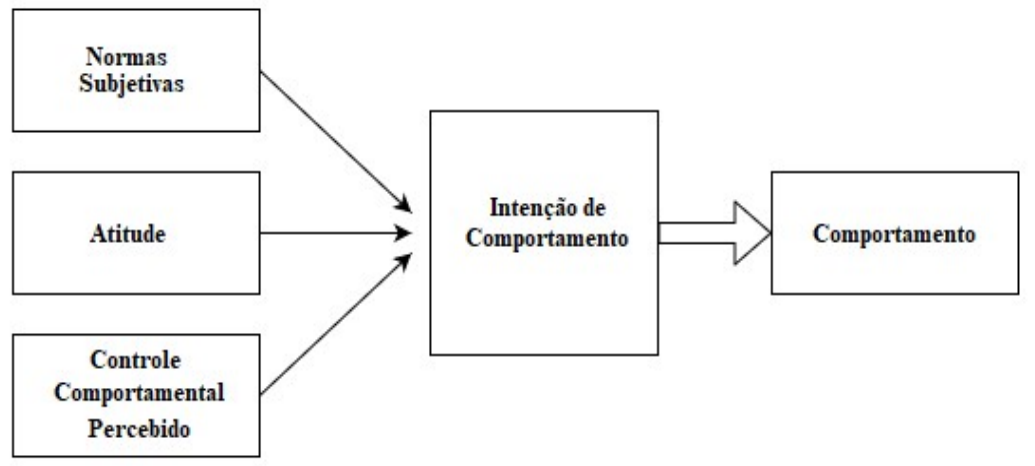

Figura 2 - Teoria do Comportamento Planejado.

Fonte: adaptado de Ajzen (1991).

No que diz respeito à capacidade explicativa dessa teoria, pontua-se que apesar dela incluir mais uma variável visando aumentar sua capacidade de resposta, ela não consegue ultrapassar o nível de assertividade demonstrado pelo TAM no que concerne à explicação do que leva uma pessoa a adotar ou não uma tecnologia (Chu \& Chen, 2016; Rahman et al., 2017).

Admite-se que isso ocorre basicamente por causa de dois motivos principais, a teoria não foi construída para aplicação específica na área de TI e, portanto, não considera elementos que são relevantes quando se trata desse contexto, como por exemplo, a melhoria do desempenho que se espera com a adoção da tecnologia, a dificuldade ou a facilidade de uso da tecnologia, o status que se obtém com a utilização da tecnologia, dentre outros.

Além disso, a teoria foi estruturada sob a perspectiva de que a intenção se reflete no comportamento quando a decisão de executá-lo ou não seja realizada de acordo com a vontade própria do indivíduo (Ajzen, 1991) e não decorrente de uma ordem ou de uma circunstância que lhe "obrigue" a realizar tal comportamento, o que pode ocorrer no caso do uso de uma tecnologia.

\subsection{Modelo de Aceitação da Tecnologia - TAM}

O Modelo de Aceitação da Tecnologia (em inglês, Technology Acceptance Model - TAM) foi idealizado pelo Fred Davis, em 1986, como uma adaptação da Teoria da Ação Racional. O objetivo do autor era construir - com base nas ideias da TAR - uma teoria que se aplicasse à área da tecnologia da informação, ou seja, que se destinasse exclusivamente a explicar quais fatores influenciam a intenção de uso e o uso real de um sistema de informação.

Nesse sentido, a teoria desenvolvida se baseia, assim como a TAR, na influência que duas variáveis externas exercem sobre a intenção de um comportamento, no caso, o uso de uma tecnologia da informação, e seu desempenho efetivo (Marangunić \& Granić, 2015). As variáveis defendidas pelo modelo são: a utilidade percebida, a qual diz respeito ao nível de melhoria de desempenho que o indivíduo acredita que o uso de uma tecnologia pode lhe gerar, e a facilidade de uso percebida, que, por sua vez, se refere à quantidade de esforço que o indivíduo julga que necessita despender para utilizar a tecnologia em questão (Davis, 1986). O esquema apresentado na Figura 3 ilustra as relações de influências defendidas pelo modelo. 


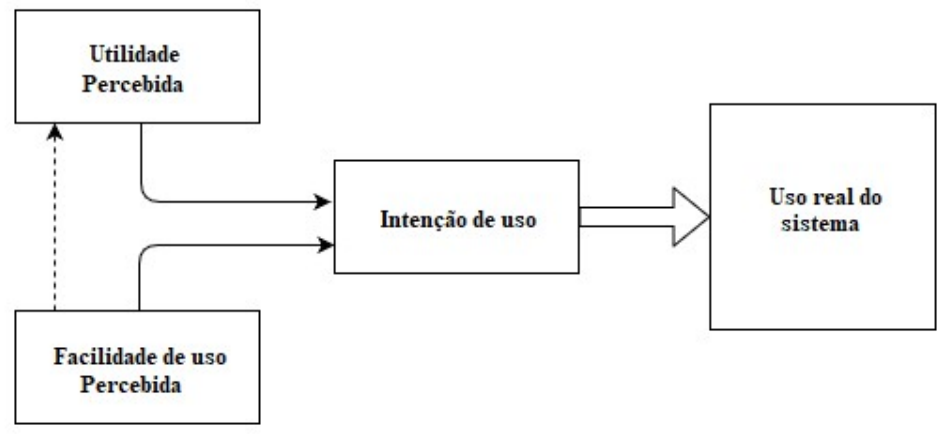

Figura 3 - Modelo de Aceitação da Tecnologia.

Fonte: adaptado de Davis (1986).

No caso do modelo em questão, apesar de sua ampla utilização em estudos que versam sobre a intenção de uso e o uso real de tecnologias, não é possível se afirmar que se trata do melhor modelo dentre os demais existentes. O mesmo foi concebido sob a hipótese de que o uso de uma tecnologia da informação é determinado principalmente pela intenção de uso que o indivíduo apresenta, e que essa intenção é definida pelas expectativas de melhoria esperada de desempenho decorrente do uso da tecnologia (utilidade percebida), e de facilidade de utilização da tecnologia (facilidade de uso percebida). Isto é, de acordo com o TAM, apenas esses dois fatores influenciam a decisão de uso do indivíduo quanto a uma tecnologia, o que não consegue abarcar completamente a multiplicidade de fatores - objetivos e subjetivos - que de fato exercem influências sobre a intenção de uso de uma tecnologia.

Um indicador que ratifica a insuficiência do TAM no que diz respeito à explicação dos fatores que determinam o uso das tecnologias é o fato de que o modelo passou por dois processos de incrementação aos longos dos anos que culminaram nos denominados TAM 2 (Venkatesh \& Davis, 2000) e TAM 3 (Venkatesh \& Bala, 2008), os quais agregaram, respectivamente, sete e treze novas variáveis à análise, conforme ilustrado pelos diagramas na Figura 4.

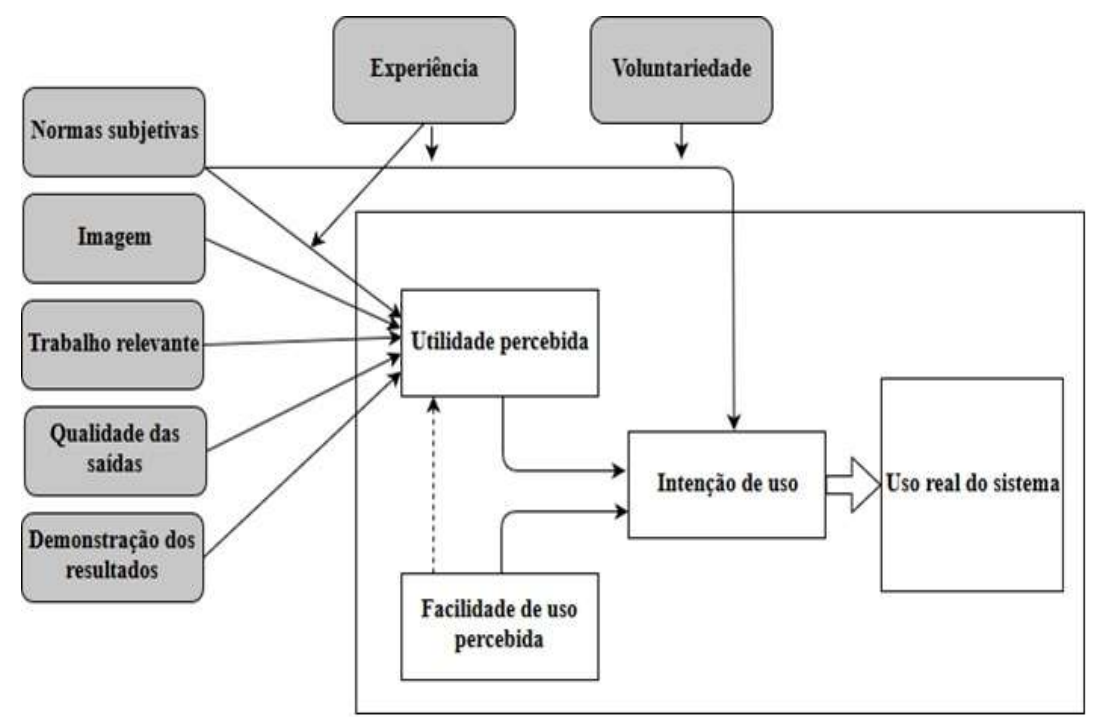

Figura 4 - Modelo de Aceitação da Tecnologia 2.

Fonte: Venkatesh \& Davis (2000).

A Figura 4 se refere ao TAM 2, também conhecido como modelo estendido, o qual inseriu sete novas variáveis na análise dos motivos que levam o indivíduo a usar ou não determinada tecnologia. Segundo os autores do modelo, ele foi testado e seus construtos foram amplamente validados, sendo significantes para a compreensão da questão estudada. O modelo disposto na Figura 5 ilustra o TAM 3, o qual desenvolveu de forma mais significativa o Modelo 
de Aceitação da Tecnologia, trazendo para a análise mais seis variáveis ainda não consideradas nas fases anteriores do TAM.

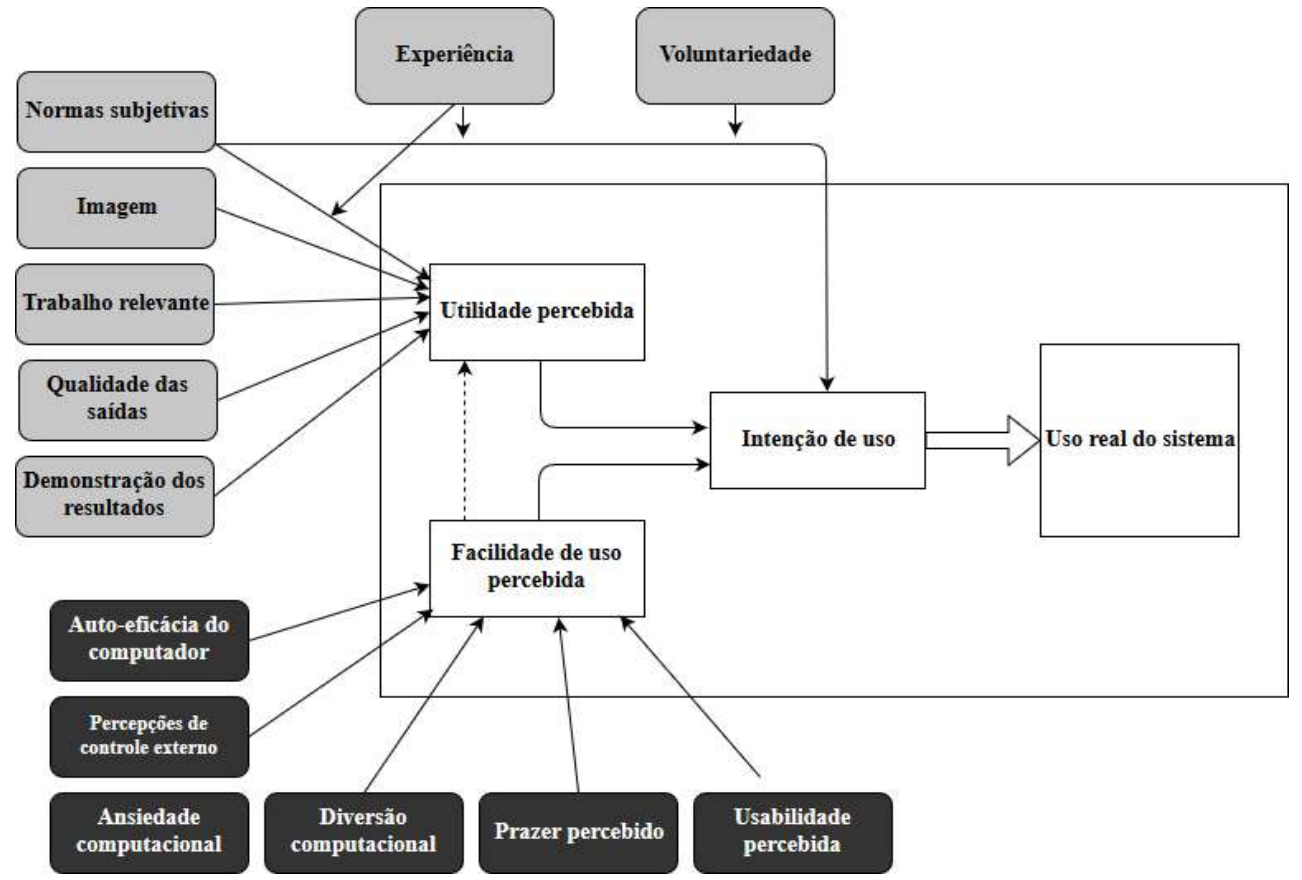

Figura 5 - Modelo de Aceitação da Tecnologia 3.

Fonte: Venkatesh e Bala (2008).

Dentre as três "faces" do TAM, entende-se que a "terceira versão" se destaca como aquela que possui maior capacidade explicativa no que diz respeito aos fatores que conduzem ao uso de uma nova tecnologia. Isso se dá principalmente em função das variáveis adicionais que são inseridas no modelo, as quais, conjuntamente, possibilitam uma análise mais rica - ao menos em termos de conteúdo - sobre a relação dos indivíduos com as tecnologias. Além disso, o TAM 3 se destaca por apresentar variáveis de cunho estritamente pessoal, o que conduz a análise para uma perspectiva mais humana, sendo o indivíduo o ator determinante da decisão de uso ou não de uma tecnologia.

Contudo, de modo geral, pontua-se que o Modelo de Aceitação da Tecnologia original apresenta-se como o mais bem-sucedido em termos de adoção (Rondan-Cataluña, Arenas-Gaitán \& Ramírez-Correa, 2015), mesmo apresentando limitações em função de outros fatores que não são considerados no modelo. Ademais, entende-se que a inclusão de novas variáveis ao TAM torna o modelo mais complicado e não gera retornos em se tratando do aumento de seu poder preditivo (Cheng, 2019).

Entre os esforços empreendidos para o desenvolvimento do TAM, Venkatesh et al. (2003) desenvolveram um novo modelo de aceitação da tecnologia, a UTAUT. Esta nova estrutura é comparável ao TAM, pois seus determinantes são similares no tocante à conceituação, apesar de ser mais difícil de ser validada em função da maior quantidade de variáveis utilizadas (Nistor \& Heymann, 2010; Scherer, Siddiq \& Tondeur, 2019). Na sequência, serão pontuadas as características e as críticas à UTAUT.

\subsection{Teoria Unificada de Aceitação e Uso de Tecnologia - UTAUT}

Esta teoria, desenvolvida por Venkatesh et al. (2003), é o resultado de uma revisão e consolidação dos construtos de oito modelos que buscam explicar o comportamento de uso de sistemas de informação, a qual tem como objetivo explicar as intenções do usuário de usar uma tecnologia da informação e o seu comportamento de uso subsequente. A Teoria Unificada de Aceitação e Uso de Tecnologia, ou como é conhecida: UTAUT, trata-se de um modelo unificado com quatro determinantes centrais de intenção de uso e quatro moderadores de relaçõeschave, os quais em conjunto fornecem uma ferramenta útil para a avaliação da probabilidade de sucesso de novas tecnologias (Venkatesh et al., 2003).

Dentre as quatro variáveis principais do modelo, destaca-se que a expectativa de desempenho diz respeito ao grau em que um indivíduo acredita que o uso da tecnologia irá ajudá-lo a obter ganhos no desempenho do trabalho; 
A expectativa de esforço se refere ao grau de facilidade associada ao uso do sistema; A influência social pode ser compreendida como o grau em que um indivíduo percebe que outras pessoas acreditam que ele deve usar o novo sistema; E as condições facilitadoras são entendidas como o grau em que um indivíduo acredita que existe uma infraestrutura organizacional e técnica para apoiar o uso do sistema (Venkatesh et al., 2003).

As outras quatro variáveis moderadoras têm a função de controlar o efeito das variáveis principais na questão de interesse, isto é, elas atuam como uma espécie de restrição, não permitindo que as outras variáveis sejam ponderadas de maneira equitativa para todos os indivíduos analisados. Em outras palavras, as variáveis gênero, idade, experiência e voluntariedade possibilitam verificar o impacto de cada uma das variáveis principais na intenção de uso considerando as características específicas dos indivíduos (Venkatesh et al., 2003). O esquema da Figura 6 demonstra as relações defendidas pelo modelo.

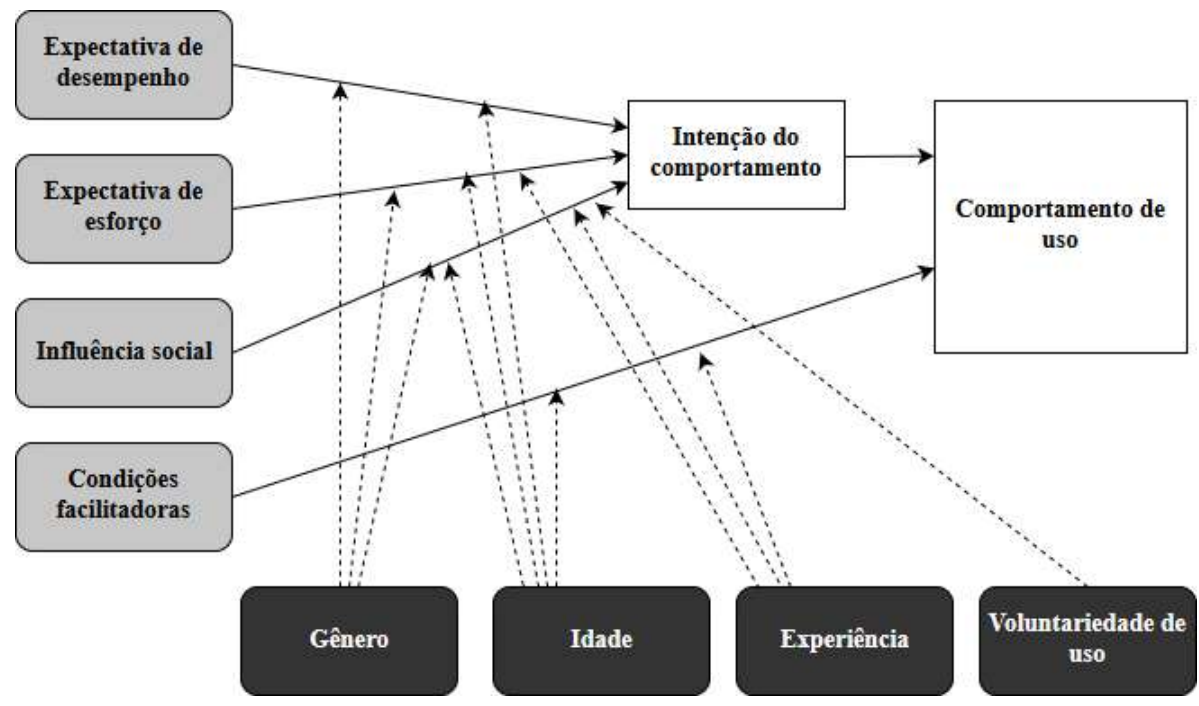

Figura 6 - Teoria Unificada de Aceitação e Uso de Tecnologia.

Fonte: Venkatesh et al. (2003).

Conforme os autores, o modelo foi testado e obteve validação de seus construtos, respondendo em aproximadamente $70 \%$ à questão dos fatores que influenciam a intenção de uso e o uso subsequente de uma tecnologia (Venkatesh et al., 2003). Outros trabalhos (Dulle \& Minishi-Majanja, 2011; Meirelles \& Longo, 2014; Rahman et al., 2017) também comprovam a eficácia da UTAUT.

Os resultados da validação do modelo demonstraram a dinâmica relação existente entre as variáveis e comprovaram que uma variável não pode ser analisada isoladamente quando se trata da área de tecnologias da informação, pois cada variável principal é influenciada direta ou indiretamente pelas moderadoras, isto é, pelas características pessoais de cada indivíduo. Além disso, estudos realizados também demonstraram que o percentual de explicação de cada variável do modelo varia conforme o contexto e as especificidades de cada tecnologia de informação analisada (Gupta, Dasgupta \& Gupta, 2008; Li \& Kishore, 2006; Rahman et al., 2017)

Nesse sentido, destaca-se que a UTAUT se apresenta como um modelo de aceitação da tecnologia com alta capacidade explicativa. Porém também não se pode dizer que se trata de um modelo ideal, tendo em vista que não há um consenso quanto aos níveis de resposta das suas variáveis (Rahman, et al., 2017). Assim sendo, pondera-se que mesmo esse modelo, com um maior número de variáveis, não consegue responder de forma contundente todos os fatores que exercem influência sobre o indivíduo na tomada de decisão quanto ao uso (ou não) de uma tecnologia da informação.

Esse fato decorre principalmente de algumas disfunções encontrada no modelo, como, por exemplo: o fato dele propor uma análise estritamente focada na percepção do indivíduo e não considerar variáveis externas que exercem influência sobre ele; A visão pró-tecnologia defendida indiretamente pelo modelo, isto é, parte-se da premissa de forma indireta que o indivíduo está disposto a aceitar a tecnologia e o que se busca com a análise é somente verificar quais os principais fatores que influenciam essa adoção; e, por fim, a subjetividade excessiva das principais variáveis, o que pode gerar vieses à análise e ao entendimento da questão em estudo.

\subsection{Síntese analítica}


Os modelos apresentados detêm uma diversidade de características que denotam semelhanças e dessemelhanças entre eles. Buscando sintetizar tais características e apresentar a fundamentação lógica das críticas tecidas para cada um deles, elaborou-se o Quadro 1.

Quadro 1 - Síntese analítica dos modelos de aceitação da tecnologia.

\begin{tabular}{|c|c|c|}
\hline Modelo & Variáveis observadas & Críticas \\
\hline \multirow{2}{*}{$\begin{array}{l}\text { Teoria da Ação } \\
\text { Racional }\end{array}$} & Normas Subjetivas & \multirow{2}{*}{$\begin{array}{l}\text { A teoria parte do pressuposto que os indivíduos tomam } \\
\text { decisões quanto aos seus comportamentos a partir de uma } \\
\text { análise racional, o que não pode ser concebido quando se } \\
\text { aplica a decisão de uso ou não de uma tecnologia, dado a } \\
\text { existência de variáveis subjetivas que exercem influência } \\
\text { sobre o indivíduo nesse contexto. }\end{array}$} \\
\hline & Atitude & \\
\hline \multirow{3}{*}{$\begin{array}{l}\text { Teoria do } \\
\text { Comportamento } \\
\text { Planejado }\end{array}$} & Normas Subjetivas & \multirow{3}{*}{$\begin{array}{l}\text { A TPB não considera elementos relevantes quando se } \\
\text { trata da decisão de comportamento diante de uma } \\
\text { tecnologia, e a teoria parte do pressuposto que o } \\
\text { comportamento realizado pelo indivíduo decorre tão } \\
\text { somente de sua vontade individual, não considerando } \\
\text { fatores extrínsecos que exercem influência sobre ele. }\end{array}$} \\
\hline & Atitude & \\
\hline & $\begin{array}{l}\text { Controle comportamental } \\
\text { percebido }\end{array}$ & \\
\hline \multirow{2}{*}{$\begin{array}{l}\text { Modelo de } \\
\text { Aceitação da } \\
\text { Tecnologia }\end{array}$} & Utilidade Percebida & \multirow{2}{*}{$\begin{array}{l}\text { Para o TAM, somente duas variáveis exercem influência } \\
\text { sobre a decisão de uso do indivíduo quanto a uma } \\
\text { tecnologia, o que é insuficiente para explicar esse } \\
\text { processo, dado a quantidade de outros fatores - objetivos } \\
\text { e subjetivos - que influenciam o indivíduo nessa decisão. }\end{array}$} \\
\hline & Facilidade de uso & \\
\hline \multirow{5}{*}{$\begin{array}{l}\text { Modelo de } \\
\text { Aceitação da } \\
\text { Tecnologia } 2 \\
\text { (além das } \\
\text { variáveis do } \\
\text { TAM) }\end{array}$} & Normas subjetivas & \multirow{11}{*}{$\begin{array}{l}\text { Apesar dessas outras duas faces do TAM incluírem novas } \\
\text { variáveis e buscarem realizar uma explicação mais } \\
\text { profunda, elas não conseguem obter um maior grau de } \\
\text { capacidade explicativa, isso se deve, possivelmente, a } \\
\text { pouca significância das variáveis inseridas no modelo } \\
\text { para a decisão de uso de uma tecnologia, que é a variável } \\
\text { de interesse. }\end{array}$} \\
\hline & Imagem & \\
\hline & Trabalho relevante & \\
\hline & Qualidade das saídas & \\
\hline & Demonstração dos resultados & \\
\hline \multirow{6}{*}{$\begin{array}{l}\text { Modelo de } \\
\text { Aceitação da } \\
\text { Tecnologia } 3 \\
\text { (além das } \\
\text { variáveis do TAM } \\
\text { e do TAM 2) }\end{array}$} & Auto-eficácia do computador & \\
\hline & $\begin{array}{l}\text { Percepções de controle } \\
\text { externo }\end{array}$ & \\
\hline & Ansiedade computacional & \\
\hline & Diversão computacional & \\
\hline & Prazer percebido & \\
\hline & Usabilidade percebida & \\
\hline \multirow{8}{*}{$\begin{array}{l}\text { Teoria Unificada } \\
\text { de Aceitação e } \\
\text { Uso de } \\
\text { Tecnologia }\end{array}$} & Expectativa de desempenho & \multirow{8}{*}{$\begin{array}{l}\text { O modelo defende uma análise estritamente focada na } \\
\text { percepção do indivíduo; sustenta indiretamente uma } \\
\text { visão pró-tecnologia, isto é, parte-se da premissa que o } \\
\text { indivíduo está disposto a aceitar a tecnologia; e considera } \\
\text { muitas variáveis subjetivas que não permitem } \\
\text { compreender a totalidade dos fatores que influenciam o } \\
\text { indivíduo. }\end{array}$} \\
\hline & Expectativa de esforço & \\
\hline & Influência social & \\
\hline & Condições facilitadoras & \\
\hline & Idade & \\
\hline & Gênero & \\
\hline & Experiência & \\
\hline & Voluntariedade de uso & \\
\hline
\end{tabular}

Fonte: Elaboração própria (2019). 
A despeito das críticas realizadas, os modelos de aceitação da tecnologia existentes possuem validade no tocante ao rigor com o qual foram construídos e à finalidade a que se propõem, não sendo o caso de baixa qualificação ou capacidade teórica por parte dos pesquisadores que os desenvolveram, tampouco dos demais que se debruçam sobre o tema. O ponto que se estabelece é a complexa variedade de fatores que rodeiam o indivíduo e que exercem influência na decisão de uso de uma tecnologia da informação. Nesse sentido, evidencia-se que a baixa possibilidade da existência de um modelo que abarque todos os fatores que exercem influência sobre a decisão de uso de uma tecnologia, o que ocorre principalmente em função de que não se pode estabelecer algo de forma objetiva quando se trata de analisar os fatores que influenciam subjetivamente a decisão de uma pessoa.

\section{Considerações Finais}

Este trabalho buscou fazer uma análise crítica acerca dos principais modelos de aceitação da tecnologia existentes, os quais procuram explicar quais as principais variáveis que influenciam a intenção de uso de uma tecnologia por parte do usuário. Para tanto, realizou-se uma breve exposição dos modelos: Teoria da Ação Racional; Teoria do Comportamento Planejado; Modelo de Aceitação da Tecnologia e suas versões; e a Teoria Unificada de Aceitação e Uso de Tecnologia, em conjunto com uma análise crítica acerca dos construtos de cada um deles.

Tais críticas possibilitaram o entendimento de que todos os modelos discutidos conseguem explicar em algum nível a questão de pesquisa. Todavia nenhum deles possui capacidade explicativa em termos de conseguir desvendar todos os fatores que exercem influência sobre a decisão de uso ou não de uma tecnologia por parte do indivíduo. $\mathrm{O}$ que se acredita que ocorre em função da pluralidade de variáveis que rodeiam o indivíduo e que exercem influência sobre essa decisão de uso.

De modo geral, acredita-se que os modelos apresentados são eficazes no que se propõem e que as limitações existentes são decorrentes da complexidade do fenômeno que eles buscam tratar. Sendo pouco provável a existência de um modelo ótimo capaz de responder de forma a questões sobre quais os fatores que influenciam o uso de uma tecnologia da informação. Pontua-se também que a baixa probabilidade desse "modelo ideal" também se deve ao fato que cada processo de adoção e uso de uma tecnologia é único e influenciado por variáveis específicas que afetam o indivíduo de forma particular.

Ademais, acrescenta-se como possíveis futuros trabalhos decorrentes deste ensaio: o aprofundamento das críticas ora expostas, o que pode ser concebido mediante aplicações empíricas dos modelos, e a realização de trabalhos com outras metodologias que permitam fundamentar de modo mais amplo os modelos apresentados.

Por fim, destacam-se as contribuições teóricas deste ensaio para o crescimento da literatura nacional sobre tecnologias da informação, principalmente no que diz respeito aos fatores que exercem influência sobre a decisão de adoção e uso dessas tecnologias. Como implicações práticas, reconhece-se a necessidade da realização de estudos em âmbito nacional que validem os modelos apresentados como forma de proporcionar um maior nível de assertividade para o planejamento de tecnologias.

\section{Referências}

Ajzen, I. (1991). The Theory of Planned Behavior. Organizational Behavior and Human Decision Processes, San Diego (CA), 50, 179-211. https://doi.org/10.1016/0749-5978(91)90020-T

Ajzen, I. (2011). The theory of planned behaviour: reactions and reflections, Psychology and Health, v. 26, n. 9, p. 1113-1127, 2011. https://doi.org/10.1080/08870446.2011.613995

Boava, D. L. T., Macedo, F. M. F., \& Sette, R. de S. (2012, maio). Contribuições do ensaio teórico para os estudos organizacionais. Curitiba/PR. Anais do VII Encontro de Estudos Organizacionais da Anpad, Curitiba, PR, Brasil, 7. http://www.anpad.org.br/eventos.php?cod_evento $=4 \&$ cod_evento_edicao $=61 \&$ cod_edicao_subse $\mathrm{cao}=812 \&$ cod_edicao_trabalho $=14247$

Bobsin, D., Visentini, M. S., \& Rech, I. (2009). Em busca do estado da arte da UTAUT: ampliando as considerações sobre o uso da tecnologia. Revista de Administração e Inovação, 6(2), 99-118. http://www.revistas.usp.br/rai/article/view/79142/83214 
Bouwman, H., Van Den Hooff, B., Van De Wijngaert, L., \& Van Dijk, J. (2005). Information and communication technology in organizations: adoption, implementation, use and effects. Sage. http://dx.doi.org/10.4135/9781446211519

Carvalho, M. L. A. de, Freitas, A. S. de, Ramos, A. S. M., Nascimento, T. C., Ferreira, J. B., (2013). Fatores que afetam a intenção em continuar o uso do e-learning: um estudo com professores de uma universidade federal. Revista Administração em Diálogo, 15(1), 139-164. https://doi.org/10.20946/rad.v15i1.12151

Cheng, E. W. (2019). Choosing between the theory of planned behavior (TPB) and the technology acceptance model (TAM). Educational Technology Research and Development, 67(1), 21-37. https://doi.org/10.1007/s11423-018-9598-6

Chu, T. H., \& Chen, Y. Y. (2016). With good we become good: Understanding e-learning adoption by theory of planned behavior and group influences. Computers \& Education, 92, 37-52. https://doi.org/10.1016/j.compedu.2015.09.013

Davis, F. D. (1989). Perceived of use, and user acceptance of information technology. MIS Quartelly, Minneapolis, USA, 13(3), 319-338. https://doi.org/10.2307/249008

Davis, F. D., Bagozzi, R., \& Warshaw, P. R. (1989). User acceptance of computer technology: a comparison of two theoretical models. Management Science, 5(8), 982-1003. https://doi.org/10.1287/mnsc.35.8.982

Davis, F. D. (1986). A technology acceptance model for empirically testing new end-user information systems: theory and results dissertation). https://www.researchgate.net/publication/35465050_A_Technology_Acceptance_Model_for_Empirically_ Testing_New_End-User_Information_Systems

Dulle, F. W., \& Minishi-Majanja, M. K. (2011). The suitability of the Unified Theory of Acceptance and Use of Technology (UTAUT) model in open access adoption studies. Information development, 27(1), 32-45. https://doi.org/10.1177/0266666910385375

Fishbein, M. \& Ajzen, I. (1975). Belief, Attitude. Intention and Behavior: An Introduction to Theory and Research. Reading, MA: Addison-Wesley Publishing. https://www.researchgate.net/publication/233897090_Belief_attitude_intention_and_behaviour_An_introdu ction_to_theory_and_research

Gupta, B., Dasgupta, S., \& Gupta, A. (2008). Adoption of ICT in a government organization in a developing country: An empirical study. The Journal of Strategic Information Systems, 17(2), 140-154. https://doi.org/10.1016/j.jsis.2007.12.004

Lai, P. C. (2017). The literature review of technology adoption models and theories for the novelty technology. JISTEM-Journal of Information Systems and Technology Management, 14(1), 21-38. http://dx.doi.org/10.4301/S1807-17752017000100002

Li, J. P., \& Kishore, R. (2006, April). How robust is the UTAUT instrument?: a multigroup invariance analysis in the context of acceptance and use of online community weblog systems. In Proceedings of the $2006 \mathrm{ACM}$ SIGMIS CPR conference on computer personnel research: Forty-four years of computer personnel research: achievements, challenges \& the future (pp. 183-189). ACM. https://doi.org/10.1145/1125170.1125218

Marangunić, N., \& Granić, A. (2015). Technology acceptance model: a literature review from 1986 to 2013. Universal Access in the Information Society, 14(1), 81-95. https://doi.org/10.1007/s10209-014-0348-1

Meirelles, F. de S., \& Longo, L., (2014). Adoção de plataforma estratégica de tecnologia de informação e comunicação: análise baseada no modelo UTAUT. Rev. FAE, 17(1), 110-125. https://revistafae.fae.edu/revistafae/article/view/9 
Nistor, N., \& Heymann, J. O. (2010). Reconsidering the role of attitude in the TAM: An answer to Teo (2009a). British Journal of Educational Technology, 41(6), 142-145. https://doi.org/10.1111/j.14678535.2012.01327.x

Rahman, M. M., Lesch, M. F., Horrey, W. J., \& Strawderman, L. (2017). Assessing the utility of TAM, TPB, and UTAUT for advanced driver assistance systems. Accident Analysis \& Prevention, 108, 361-373. https://doi.org/10.1016/j.aap.2017.09.011

Rondan-Cataluña, F. J., Arenas-Gaitán, J., \& Ramírez-Correa, P. E. (2015). A comparison of the different versions of popular technology acceptance models: A non-linear perspective. Kybernetes, 44(5), 788-805. https://doi.org/10.1108/K-09-2014-0184

Scherer, R., Siddiq, F., \& Tondeur, J. (2019). The technology acceptance model (TAM): A meta-analytic structural equation modeling approach to explaining teachers' adoption of digital technology in education. Computers \& Education, 128, 13-35. https://doi.org/10.1016/j.compedu.2018.09.009

Silva, P. M., \& Dias, G., (2007). Teorias sobre aceitação de tecnologia: por que os usuários aceitam ou rejeitam as tecnologias de informação? Brazilian Journal of Information Science, 1(2), 69-91. https://doi.org/10.36311/1981-1640.2007.v1n2.05.p69

Tavares, E. \& Costa, I. (2008). El papel de los valores individuales en la interacción entre los individuos y la Tecnología de la Información. Rev. Portuguesa e Brasileira de Gestão, 7(3), 11-21. http://www.scielo.mec.pt/pdf/rpbg/v7n3/v7n3a03.pdf

Venkatesh, V. \& Davis, F. D., (2000). A theoretical extension of the technology acceptance model: four longitudinal field studies. Management Science, Ann Arbor (MI), 46(2), 186-204. https://doi.org/10.1287/mnsc.46.2.186.11926

Venkatesh, V., Morris, M. G., Davis, G. B. \& Davis, F. D., (2003). User acceptance of information technology: toward a unified view. Mis Quarterly, Minneapolis (MN), 27(3), 425-478. https://doi.org/10.1287/10.2307/30036540

Venkatesh, V., \& Bala, H., (2008). Technology acceptance model 3 and a research agenda on interventions. Decision Sciences, 39(2), 273-315. https://doi.org/10.1111/j.1540-5915.2008.00192.x

Williams, M. D., Rana, N. P., \& Dwivedi, Y. K. (2015). The unified theory of acceptance and use of technology (UTAUT): a literature review. Journal of Enterprise Information Management, 28(3), 443-488. https://doi.org/10.1108/JEIM-09-2014-0088 